\title{
DETECTOR DESIGN FOR PARAMETRIC SPEECH WATERMARKING
}

\author{
A. Gurijala and J. R. Deller, Jr. \\ Michigan State University \\ Dept. Elec. \& Computer Engr. / 2120 EB \\ East Lansing, MI 48824 USA
}

\begin{abstract}
Parametric watermarking is effected by modifying the linear predictor coefficients of speech. In this work, the parameter noise is analyzed when watermarked speech is subjected to additive white and colored noise in the time domain. The paper presents two detection techniques for parametric watermarking. The first approach uses the Neyman-Pearson criterion to solve a binary decision problem. In the second approach, discriminant functions based on the minimum-error-rate criterion are used to determine which of the many watermarks was embedded or if no watermark is present. Experiments with speech data are used to determine the false-alarm and missed detection rates.
\end{abstract}

\section{INTRODUCTION}

Digital media and global access to high-speed computer networks have created complex problems for owners of intellectual property. Digital watermarking has emerged as a new technology for the protection of copyrighted material. Digital watermarking is the process of embedding data (watermark) imperceptibly into the host signal (coversignal), resulting in the stegosignal. When copyright questions arise, the watermark is recovered from the stegosignal as evidence of title. The design of a watermarking strategy involves the balancing of two principal criteria. First, embedded watermarks must be imperceptible to the listener. Second, watermarks must be robust. That is, they must be able to survive attacks- those deliberately designed to destroy or remove them, as well as distortions inadvertently imposed upon the watermarks by technical processes (e.g., compression) or by systemic processes (e.g., channel noise). Related measures of a watermark's efficacy include data payload, the number of watermark bits per unit of time [1]. Another important requirement of a watermarking strategy is its security, the inherent protection against unauthorized removal, embedding or detection [1].

This paper deals with watermark detector design for parametric watermarking of speech data. Parametric watermarking [2] is based on manipulation of linear predictor parameter values [3] of speech signals. Generally speaking, the watermark information is concentrated in the few linear prediction (LP) coefficients during the watermark embedding and recovery processes, while it is dispersed in time and spectrally otherwise.

A common approach to watermark detection involves the hypotheses, $H_{0}: I_{R}=I$ and $H_{1}: I_{R}=I+W$, where $I_{R}$ is the received signal, $I$ is the original signal and $W$ is the watermark signal [4], [5]. A Bayesian or Neyman-Pearson approach

This work was supported by the National Science Foundation of the United States under Cooperative Agreement No. IIS-9817485. Any opinions, findings, and conclusions or recommendations expressed in this material are those of the authors and do not necessarily reflect the views of the NSF. is followed in deriving the detection thresholds. For image watermarking, the image DCT coefficients are generally modeled as generalized Gaussian in distribution [4]. Most of these approaches do not consider the effect of noise while deriving the detection threshold. Several watermark detectors are based on correlation detection [6], [7]. That is, the correlation between the original and recovered watermarks or the correlation between the original watermark and recovered signal is compared against a threshold. Correlation detectors are optimal when the watermark and noise are jointly Gaussian, or in case of blind detectors the watermarked signal and noise should be jointly Gaussian. For example, the detector presented in $[1, \mathrm{Ch} .6]$ assumes that the detector output for each bit is Gaussian distributed. This is true for watermark patterns that are white, which is not the case in parametric watermarking. In this paper, watermark detection in parametric domain is considered. The stegosignal is distorted by additive white or colored Gaussian noise in the time domain. The resulting noise in the parametric domain is analyzed. Watermark detection in the parametric domain is converted into a binary or multiple decision problem in the presence of additive noise.

\section{BACKGROUND}

In the present study, the coversignal is assumed to be generated by an LP model,

$$
y_{n}=\sum_{i=1}^{M} a_{i} y_{n-i}+\xi_{n} .
$$

The "true" model is determined by standard LP analysis of a (long) frame selected for watermarking [3]. The sequence $\left\{\xi_{n}\right\}$ is the prediction residual associated with the estimated model. The stegosignal is constructed using the FIR filter model

$$
\tilde{y}_{n}=\sum_{i=1}^{M} \tilde{a}_{i} y_{n-i}+\xi_{n}
$$

where $\left\{\tilde{a}_{i}\right\}$ represents a deliberately perturbed version of the "true" set $\left\{a_{i}\right\}$. The algorithmic steps of the LP parameter-embedded watermarking procedure appear in Table 1.

The algorithm for recovering the watermark from the stegosignal appears in Table 2. An important step in the recovery process, is the least squares estimation of modified watermark coefficients. In the stegosignal, the watermark information is spread out, while during recovery the watermark information is concentrated in a few coefficients $\left\{\omega_{i}\right\}_{i=1}^{M}$ derived from an estimate of the modified LP coefficients. Parametric watermarking involves informed detection, and the coversignal is required for watermark recovery. $\mathrm{CWR}_{\text {seg }}$ is used to measure stegosignal fidelity and is defined as,

$$
\mathrm{CWR}_{\mathrm{seg}}=\frac{1}{K} \sum_{j=1}^{K} 10 \log _{10}\left[\sum_{l=k_{j}-L+1}^{k_{j}} \frac{y_{l}^{2}}{\left[\tilde{y}_{l}-y_{l}\right]^{2}}\right] \text {, }
$$


Table 1. WATERMARK EMBEDding Algorithm

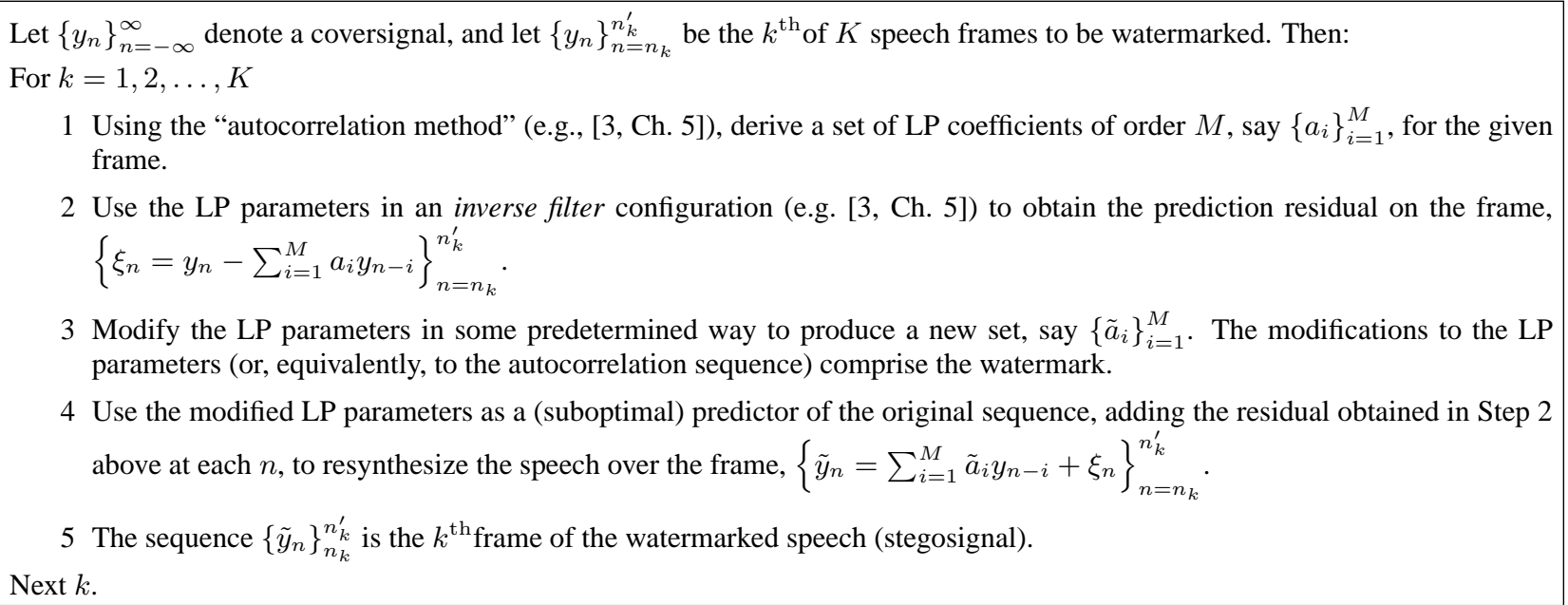

where, $k_{1}, k_{2}, \ldots, k_{K}$ are the end-times for the $K$ frames, each of which is length $L$. The $\mathrm{CWR}_{\text {seg }}$ assigns equal weight to the loud and soft portions of speech. For computing $\mathrm{CWR}_{\mathrm{seg}}$, speech frames of $15 \mathrm{~ms}$ duration were used. A simple way to control the fidelity of the stegosignal is to scale the watermark vector, $\omega$, by a constant, say $\kappa$, before adding it to the original LP parameters (Step 3 of Table 1).

\section{WATERMARK DETECTION IN PARAMETER DOMAIN}

The watermarks are comprised of non-binary orthogonal vectors of length eight. Each of the eight orthogonal vectors $\left(\omega^{k}\right.$ for $k=$ $1, \cdots, 8)$ can be interpreted as symbols from an alphabet of size eight. The watermark may be composed of many such symbols. In the examples in this paper, each orthogonal watermark vector (symbol) of length eight, is embedded into 0.125 seconds of speech, sampled at $16 \mathrm{kHz}$. The watermark vector is added to the coefficients of an eighth order linear predictor model. The length of the watermark vector (and hence the predictor model order) and the duration of speech frame can be selected quite arbitrarily, subject to certain constraints on stegosignal fidelity. These constraints include an upper limit on predictor model order, and a need to use FIR models of small order for very short speech frames ( 500 samples).

Noise in the LP domain, caused by stegosignal exposure to additive noise, can be modeled as having a Gaussian distribution. Figure 1(a) shows a typical noise distribution in the LP domain when white noise (SNR $15 \mathrm{~dB}$ ) is added to the stegosignal. The noise distribution for Fig. 1(a) was obtained by conducting 1000 experiments, involving a stegosignal of $1 \mathrm{~s}$ duration watermarked at $\mathrm{CWR}_{\text {seg }}$ of $7 \mathrm{~dB}$ using a watermark message consisting of eight orthogonal vectors, each vector embedded into 0.125 seconds or 2000 samples of speech. The coversignal was the sentence from TIMIT database [9], "She had your dark suit in greasy wash water all year," sampled at $16 \mathrm{kHz}$. When white Gaussian noise was added to the stegosignal, the noise samples affecting a particular watermark coefficient, in the parameter domain were uncorrelated and could be approximated as independent and identically distributed (i.i.d) Gaussian noise. The LP noise affecting a watermark coefficient was uncorrelated with the LP noise affecting a different watermark coefficient. The noise samples were also uncorrelated with the corresponding LP coefficients. It should be noted that when the stegosignal is subjected to white Gaussian noise, the parameter noise is of very low power and asymptotically tends to zero. However, the noise generated using the "randn" function in matlab, is not ideal white noise.

The parametric noise distribution when the stegosignal was affected by colored noise is similar to that shown in Fig. 1(b). Colored noise was generated by filtering a white noise process using a $11^{\text {th }}$ order FIR lowpass filter with a cut-off frequency of $6400 \mathrm{~Hz}$. The LP noise affecting any given watermark coefficient was found to be Gaussian and i.i.d. in nature. However, a realization of noise affecting all the watermark coefficients was found to be correlated with the original LP coefficients. A possible solution to this problem is to normalize the watermark coefficients before adding them to the original LP coefficients. That is, instead of directly adding the watermark vector to the original LP coefficients $(\tilde{\mathbf{a}}=\mathbf{a}+\omega$, where $\omega$ is any of the eight orthogonal vectors $\omega^{k}$ ), we obtain the modified LP coefficients as,

$$
\tilde{a}_{i}=a_{i}+\omega_{i}\left|a_{i}\right| .
$$

From the estimate of the modified LP coefficients, the watermark

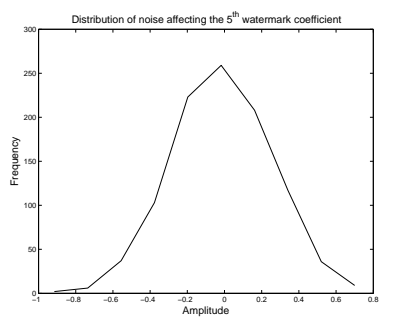

(a)

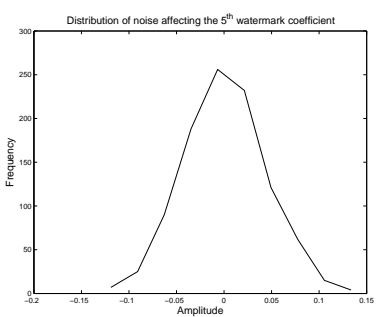

(b)
Fig. 1. Noise distribution in the LP domain.

vector vector is obtained as,

$$
\hat{\omega}_{i}=\frac{\hat{\tilde{a}}_{i}-a_{i}}{\left|a_{i}\right|}
$$

with $\hat{\tilde{\mathbf{a}}}=\left\{a_{i}\right\}_{i=1}^{M}$, as defined in Table 2. However, when $\left|a_{i}\right| \ll$ 1 , the recovery of watermark coefficients magnifies the noise variance in the LP domain. To avoid this, watermark coefficients are 
Table 2. WATERMARK ReCOVERY AlgORITHM

For $k=1,2, \ldots, K$

1 Subtract residual frame $\left\{\xi_{n}\right\}_{n_{k}}^{n_{k}^{\prime}}$ from the stegosignal frame $\left\{\tilde{y}_{n}\right\}_{n_{k}}^{n_{k}^{\prime}}$. This results in an estimate of the modified predicted speech, $\left\{d_{n}=\tilde{y_{n}}-\xi_{n}\right\}_{n_{k}}^{n_{k}^{\prime}}$.

2 Estimate the modified LP coefficients $\left\{\tilde{a}_{i}\right\}_{1}^{M}$ by computing the least-square-error solution, say $\left\{\hat{\tilde{a}}_{i}\right\}_{1}^{M}$, to the overdetermined system of equations: $d_{n} \approx \sum_{i=1}^{M} \alpha_{i} y_{n-i}, n=n_{k}, \ldots, n_{k}^{\prime}$.

3 Use the parameter estimates from Step 2 to derive the corresponding watermark values.

Next $k$.

Table 3. EFFECT OF SELECTIVE NORMALIZATION

\begin{tabular}{|c|c|c|c|c|c|}
\hline Noise & SNR (dB) & Normalization & $\mu$ & $\sigma^{2}$ & $c_{\text {ra }}(0)$ \\
\hline White & 10 & no & $2.849 \times 10^{-4}$ & 0.0517 & -0.0059 \\
\hline White & 10 & complete & -0.0152 & 4.6477 & -0.0051 \\
\hline White & 10 & selective & $-6.2 \times 10^{-5}$ & 0.1099 & $7.6445 \times 10^{-4}$ \\
\hline Colored & 15 & no & $2.3438 \times 10^{-4}$ & 0.0049 & 0.0328 \\
\hline Colored & 15 & complete & 0.0139 & 0.7518 & -0.0094 \\
\hline Colored & 15 & selective & $-1.162 \times 10^{-4}$ & 0.0071 & 0.0023 \\
\hline
\end{tabular}

normalized before embedding only if $\left|a_{i}\right| \geq 1$. For the experiments presented in the rest of the paper, the watermark embedding and recovery involves this "selective normalization." Accordingly, Step 3 of Table 1 is carried out using the following rule in the algorithm implemented here:

$$
\tilde{a}_{i}= \begin{cases}a_{i}+\omega_{i}\left|a_{i}\right|, & \text { if }\left|a_{i}\right| \geq 1 \\ a_{i}+\omega_{i}, & \text { otherwise }\end{cases}
$$

The final step in the recovery algorithm (Table 2) involves the following equation:

$$
\hat{\omega}_{i}= \begin{cases}\frac{\hat{\tilde{a}}_{i}-a_{i}}{\left|a_{i}\right|}, & \text { if }\left|a_{i}\right| \geq 1 \\ \hat{\tilde{a}}_{i}-a_{i}, & \text { otherwise }\end{cases}
$$

It is observed from Table 3 that selective normalization of watermark coefficients significantly reduces the correlation between noise in LP domain and the LP coefficients, especially when the stegosignal is subjected to colored noise in the time domain. Moreover, as the noise variance in the LP domain is reduced, selective normalization improves the correlation coefficient between the original and recovered watermarks.

\subsection{Neyman-Pearson based watermark detector}

A Neyman-Pearson solution to watermark detection is applicable to the binary decision problem of determining whether a particular watermark vector is present or not in the received signal in the presence of additive noise. Preliminary watermark detection experiments are used to set the hypotheses,

$$
\begin{gathered}
H_{0}: r_{i}=v_{i}, i=1,2, \ldots, L \\
H_{1}: r_{i}=\omega_{i}+v_{i}, i=1,2, \ldots, L
\end{gathered}
$$

where $\left\{r_{i}\right\}_{i=1}^{L}$ is the observation vector. The null hypothesis is that no watermark is present and only noise is transmitted $\left\{v_{i}\right\}_{i=1}^{L}$, while under $H_{1}$ both watermark $\left\{\omega_{i}\right\}_{i=1}^{L}$ and noise samples $\left\{v_{i}\right\}_{i=1}^{L}$, are present in additive combination. Due to selective normalization of watermark coefficients, the noise in LP domain, $v_{i}$ is approximately distributed as $\mathcal{N}\left(0, \sigma^{2}\right)$, when noise $\left\{\zeta_{i}\right\}_{i=1}^{N}$ is added to the stegosignal in the time domain such that the SNR is $S_{1}=$ $10 \log _{10} \frac{\sum_{n=1}^{N} \tilde{y}_{n}^{2}}{\sum_{n=1}^{N} \zeta_{n}^{2}}$. For this watermark detection problem, the expressions for false-alarm, detection and missed detection rates are well-known and are given by [8],

$$
\begin{gathered}
P_{F}=0.5\left[\operatorname{erfc}\left(\frac{\ln \eta+\sum_{i=1}^{L} \frac{\omega_{i}^{2}}{2 \sigma^{2}}}{\sqrt{2} \bar{\sigma}}\right)\right] \\
P_{D}=0.5\left[\operatorname{erfc}\left(\frac{\ln \eta+\sum_{i=1}^{L} \frac{\omega_{i}^{2}}{2 \sigma^{2}}-\overline{\mu_{1}}}{\sqrt{2} \bar{\sigma}}\right)\right] \\
P_{M}=1-P_{D}
\end{gathered}
$$

Here, $\overline{\mu_{1}}=\sum_{i=1}^{L} \frac{\omega_{i}^{2}}{\sigma^{2}}, \bar{\sigma}=\sqrt{\sum_{i=1}^{L} \frac{\omega_{i}^{2}}{\sigma^{2}}}$ and $\eta$ is the detection threshold. Let $\eta^{\prime \prime}=\ln \eta+\sum_{i=1}^{L} \frac{\omega_{i}^{2}}{2 \sigma^{2}}$ then, the decision rule is

$$
\sum_{i=1}^{L} \frac{r_{i} \omega_{i}}{\sigma^{2}} \underset{H_{0}}{\stackrel{H_{1}}{<}} \eta^{\prime \prime}
$$

In a practical implementation, the threshold $\tau^{\prime \prime}$, corresponding to an SNR of $S_{1}$, can be adjusted further if the actual SNR in the time domain is determined. As an example, if the SNR was found to be $S_{2}=10 \log _{10} \frac{\sum_{n=1}^{N} \tilde{y}_{n}^{2}}{\sum_{n=1}^{N} \tilde{\zeta}_{n}^{2}}$ (assuming zero-mean noise), the threshold $\tau^{\prime \prime}$ is altered by multiplying $\sigma^{2}$ with the adjustment factor $1 / \beta$, where $\beta=10^{\left(\frac{S_{1}-S_{2}}{10}\right)}$.

The SNR in the parametric domain is defined as, $d^{2}=\left(\frac{\overline{\mu_{1}}}{\bar{\sigma}}\right)^{2}[8]$. In the present case, $d=\sqrt{\overline{\mu_{1}}}$. Hence embedded marks of greater energy will result in improved robustness, while noise of higher variance in the parametric domain will hinder watermark detection. The stegosignal was subjected to white and colored noise, resulting in different SNRs in the time and parametric domain. In each case, experiments were repeated 1000 times in order to estimate the mean and variance of the Gaussian noise affecting each watermark coefficient. The receiver operating characteristics 
Table 4. Estimates of SNR, $d^{2}, P_{D}$ AND $P_{F}$

\begin{tabular}{|c|c|c|c|c|}
\hline Noise & $\mathbf{d}^{\mathbf{2}}$ & $\mathbf{P}_{\mathbf{D}}$ & $\mathbf{P}_{\mathbf{F}}$ & $\tau^{\prime \prime}$ \\
\hline White $(15 \mathrm{~dB})$ & 696.95 & 0.99999 & $4.37 \times 10^{-114}$ & 6.8699 \\
\hline White $(10 \mathrm{~dB})$ & 72.79 & 0.99994 & $1.37 \times 10^{-6}$ & 4.3960 \\
\hline Color $(7 \mathrm{~dB})$ & 167.29 & 0.99999 & $1.20 \times 10^{-18}$ & 5.4038 \\
\hline White $(3 \mathrm{~dB})$ & 14.45 & 0.9987 & 0.215 & 1.6610 \\
\hline White (1dB) & 9.54 & 0.99715 & 0.37304 & 0.8388 \\
\hline
\end{tabular}

(ROC) were determined using equations (6) and (7). It can be observed from Table 4 that the very low false-positive rates can be determined using parametric watermarking with selective normalization. For example, when $10 \mathrm{~dB}$ white noise is added to the stegosignal, for a threshold $\tau^{\prime \prime}=4.3960$, a $P_{D}=0.99999$ and a false-alarm rate $P_{F}=1.37 \times 10^{-6}$ is obtained. Experiments were performed for time domain SNRs of $1 \mathrm{~dB}$ and $3 \mathrm{~dB}$ and $P_{F}$ was found to be 0.14 and 0.0033 respectively, an improvement over the results in Table 4.

\subsection{Discriminant functions for parametric watermark detec- tion}

Discriminant functions [10] are used to assign the recovered vector to one of the many possible classes. In the present applications, there are nine classes which include the eight orthogonal watermarks and the case of no watermark being present. Discriminant functions are derived based on minimum-error-rate classification [10] for all possible watermarks and also for no watermark being present in the received signal. The recovered vector is assigned to the class that results in the highest discriminant function.

Detection is done on a per frame basis. From the previous section, it is known that the parameter noise affecting a watermark coefficient when colored noise is added in the time domain, can be approximated by $\mathcal{N}\left(0, \sigma^{2}\right)$. The noise distribution was the same even when no watermark was embedded. Since vectors of dimension eight were considered, the noise affecting all the eight coefficients of the recovered vector for a particular class $k$ can be expressed by the general multivariate normal density,

$$
p_{k}(\mathbf{r})=\frac{1}{(2 \pi)^{(4)}|\mathbf{\Sigma}|^{\frac{1}{2}}} \exp \left[-\frac{1}{2}\left(\mathbf{r}-\mu_{k}\right)^{\prime} \boldsymbol{\Sigma}^{-1}\left(\mathbf{r}-\mu_{k}\right)\right]
$$

where $\boldsymbol{\Sigma}=\sigma^{2} \mathbf{I}, \mathbf{I}$ being the identity matrix, and $\mu_{k}$ is the mean of the multivariate normal density for class $k$. For this application, $k$ takes values from 1 to 9 , with 9 corresponding to the no noise case. Hence, $\mu_{k}=\omega^{k}$ for $k=1, \cdots, 8$ and $\mu_{k=9}=\mathbf{0}$, with $\mathbf{0}$ being the zero vector. The discriminant function for class $k$ is given by,

$$
\mathbf{g}_{k}(\mathbf{r})=-\frac{1}{2 \sigma^{2}}\left[\mathbf{r}^{\prime} \mathbf{r}-2 \mu_{\mathbf{k}}^{\prime} \mathbf{r}+\mu_{\mathbf{k}}^{\prime} \mu_{\mathbf{k}}\right]
$$

The recovered vector $\mathbf{r}$ is assigned to the class the $j$ satisfying the following equation.

$$
\mathbf{g}_{j}(\mathbf{r})>\mathbf{g}_{k}(\mathbf{r}) \text { for all } j \neq k .
$$

Thus using the above expression, a watermark present in the recovered vector is detected. Experiments were performed to determine the false-alarm and detection rates and the results are tabulated in Table 5. It should be noted that for time domain SNRs below $15 \mathrm{~dB}$, the resulting stegosignals are highly noisy and are of
Table 5. Minimum-ERROR-RATE DETECTION USING DISCRIMINANT FUNCTIONS

\begin{tabular}{|c|c|c|c|}
\hline Noise & SNR $(\mathrm{dB})$ & $\mathbf{P}_{\mathbf{D}}$ & $\mathbf{P}_{\mathbf{F}}$ \\
\hline White & $3 \mathrm{~dB}$ & 0.9289 & 0.2487 \\
\hline White & $7 \mathrm{~dB}$ & 0.9939 & 0.0797 \\
\hline White & $10 \mathrm{~dB}$ & 0.9997 & 0.0198 \\
\hline White & $15 \mathrm{~dB}$ & 0.9998 & $10 \times^{-5}$ \\
\hline
\end{tabular}

no commercial significance. However, because of the low falsealarm rates $\left(<10^{-6}\right)$, it is not feasible to experimentally determine $P_{F}$ for SNRs above $15 \mathrm{~dB}$. In most watermarking applications, it is necessary to have very low false-alarm rates. Instead of using minimum-error-rate criterion where both missed detection and false-alarm are equally costly, the false-alarm error can be weighted more heavily and the discriminant functions derived accordingly.

\section{CONCLUSIONS}

Parameter noise is modeled when the stegosignal is subjected to additive white and colored noise in the time domain. Selective normalization of watermark coefficients is proposed. By using a Neyman-Pearson detector and minimum-error-rate classification, the false-alarm and missed detection rates are determined.

\section{REFERENCES}

[1] I.J. Cox, M.L. Miller and J.A. Bloom, Digital Watermarking, Academic Press, 2002.

[2] A. Gurijala, J.R. Deller, JR., M.S. Seadle and J.H.L. HANSEN, "Speech watermarking through parametric modeling," Proceedings of International Conference on Spoken Language Processing (ICSLP) (on CD-ROM), Denver, Sep. 2002.

[3] J.R. Deller, JR., J.H.L. HAnsen and J.G. ProAKis, Discrete-Time Processing of Speech Signals (2d ed.), IEEE Press, 2000.

[4] J.J. Hernandez, M. Amado and F. Perez-Gonzalez, "DCT-domain watermarking techniques for still images: Detector performance analysis and a new structure," IEEE Transactions on Image Processing, vol. 9, no. 1, 2000.

[5] M. Barni, F. Bartolini, A.D. Rosa and A. Piva, "Optimal decoding and detection of multiplicative watermarks," IEEE Transactions on Signal Processing, vol. 51, no. 4, 2003.

[6] J.P.M.G. LinNARTZ, A.C.C. KALKer and G.F. DePOVERE, "Modeling the false-alarm and missed detection rate for electronic watermarks," Lecture Notes in Computer Science, vol. 1525, pp. 329-343, Springer-Verlag, 1998.

[7] M.L. Miller and J.A. BlooM, "Computing the probability of false watermark detection," Proceedings of the Third Workshop on Information Hiding, pp. 146-158, 1999.

[8] H.V. Poor, An Introduction to Signal Detection and Estimation (2d ed.), Springer-Verlag, 1994.

[9] P.J. PRICE, "A database for continuous speech recognition in a 1000-word domain," Proceedings of IEEE ICASSP, New York, vol. 11, pp.651-654, 1988.

[10] R. O. Duda, P. E. Hart and D. G. Stork, Pattern Classification (2d ed.), Wiley Interscience, 2001. 